hep-ph/9807335

\title{
Axion Potential, Topological Defects and CP-odd Bubbles in QCD
}

\author{
Igor Halperin and Ariel Zhitnitsky \\ Physics and Astronomy Department \\ University of British Columbia \\ 6224 Agricultural Road, Vancouver, BC V6T 1Z1, Canada \\ e-mail: higor@physics.ubc.ca \\ arz@physics.ubc.ca \\ Extended version of the talk given by $A Z$ at the Axion workshop at Gainesville, FL, \\ March 13-15,1998
}

\begin{abstract}
:
It follows on general grounds that the $\theta$ dependence in QCD is more complicated than suggested by the large $N_{c}$ approach or instanton arguments. Generically, the vacuum energy $E_{v a c}(\theta)$ is a multi-valued function of $\theta$ admitting the existence of metastable states. We discuss decays of such metastable vacua in the theory with and without the axion, and point out the potential relevance of this and related phenomena for constraining a dark matter axion. Based on the analysis of the axion potential, an idea for a new axion search experiment at RHIC is suggested. It is noted that the false vacuum decay proceeds with maximal violation of $\mathrm{CP}$, even if $\theta=0$. We further speculate that the famous Sakharov criteria for baryogenesis could be satisfied at the QCD scale.
\end{abstract}




\section{Introduction}

The axion is perhaps the most well-motivated dark matter candidate ever since the early 1980's. Originally, the axion [1] and its invisible modifications [2] has been introduced into the theory not as a dark matter candidate, but rather as the particle which solves an internal fundamental QCD problem -the strong CP problem. Twenty years later we still do not know a better solution of this problem, therefore, we should admit that the axion solution of the strong CP problem has successfully passed the timing test [3]. In this respect, unlike many other exotic particles, the axion is the unique dark matter candidate.

In the standard thermal scenario, cosmic axions can be created through the radiative decay of the axion strings [4] or due to the 'misalignment' effect [5] at the QCD phase transition, see e.g. [3]. These scenarios do not exhaust all possible mechanisms for the axion production. In particular, one more possible scenario for the axion production will be suggested below in the text. We note that constraints on the axion mass (or the coupling constant $f_{a} \sim m_{a}^{-1}$ ) are very sensitive to the specific scenario for production of the cosmic axions, inflationary model, ratio $f_{a} / T_{\text {reh }}$ (where $T_{r e h}$ is the reheating temperature of the universe at the end of inflation), etc. We restrict ourselves by considering possible constraints which follow from the QCD part of the problem.

We start with recalling some popular ansätze for the axion potential. In the 'misalignment' mechanism, after the axion mass switches on at the QCD scale, the axion field begins to coherently oscillate about the minimum at $\theta=0$. Such a picture corresponds to the simplest possible choice for the axion potential $V(a)=m_{a}^{2} a^{2} / 2$ with possible anharmonic corrections, and does not take into account the periodic properties of the axion field $a \rightarrow a+2 \pi f_{a}$. One can do better and consider potential $V(a) \sim \cos \left(a / f_{a}\right)$ motivated by instantons, which is a periodic function of $a$. One can calculate anharmonic corrections for fields near the top of the potential [6], and account for topological effects due to the appearance of domain walls [4] for this potential. All these effects are potentially extremely important, and can drastically change the whole picture of the cosmic axion production.

However, the above potentials $V(a)$ are the model expressions not derived from QCD. Moreover, they do not satisfy some general requirements of the theory, see Sect.2,3. Therefore, the corresponding calculations should be considered as qualitative estimates of possible phenomena which may happen in the development of the axion field.

We recall at this point that there is the one-to-one correspondence between the form of the axion potential $V(a)$ and the vacuum energy $E_{v a c}(\theta)$ as a function of the fundamental QCD parameter $\theta$. Indeed, the axion solution of the strong $\mathrm{CP}$ problem suggests that $\theta$ parameter in QCD is promoted to the dynamical axion field $\theta \rightarrow a(x) / f_{a}$, and the QCD vacuum energy $E_{v a c}(\theta)$ becomes the axion potential $V(a)$. Therefore, our problem of analysing $V(a)$ amounts to the study of $E_{v a c}(\theta)$ in QCD without the axion.

Recently, there has been a progress in understanding the general properties of the $\theta$ dependence in QCD [7] based on a new development [8] in supersymmetric (SUSY) theories (Sect.4 and 5). The purpose of this paper is to discuss the implications of this new understanding for the study of local and global properties of the axion potential. As for the former (Sect.6.1), we note that the temperature dependence of the axion mass (and of entire axion potential) can be related with that of the QCD vacuum quark and 
gluon condensates whose temperature dependence is understood (from lattice or model calculations). Yet, the most interesting phenomena are related to the global properties of the axion potential (Sect.6.2). As will be discussed below, the vacuum energy $E_{\text {vac }}(\theta)$ (and consequently, the axion potential $V(a)$ ) is generically multi-valued, i.e. in some parametrical regions of $\theta$ extra local minima with higher energy could exist. It may lead to the phenomenon of the false vacuum decay through bubble nucleation. This process may provide a new mechanism for the axion production, as, when the axion is present, it can be supplemented by rolling to $\theta=0$ with emission of axions. Potentially, an account of these and other related phenomena may lead to the necessity to reconsider the constraints on a dark matter axion. Based on the analysis of the axion potential, we further suggest an idea for the new axion search experiment at RHIC (Sect.7). Last but not least, we note that because CP is $100 \%$ violated in such false vacuum decays, the famous Sakharov criteria [9] for baryogenesis could be satisfied at the QCD scale (Sect.8).

\section{What is known about $E_{v a c}(\theta)$ ?}

As we already mentioned, our problem is reduced to the analysis of the $\theta$ dependence of the vacuum energy due to the exact correspondence $\theta \rightarrow a / f_{a}, \quad E_{v a c}(\theta) \rightarrow V\left(a / f_{a}\right)$. What do we know about $E_{v a c}(\theta)$ ? We know a few exact statements:

1. We know the exact Ward Identity (WI) which (near the chiral limit $m_{q} \rightarrow 0$ with equal masses ) takes the form [10]:

$$
i \int d x\left\langle 0\left|T\left\{\frac{\alpha_{s}}{8 \pi} G \tilde{G}(x) \frac{\alpha_{s}}{8 \pi} G \tilde{G}(0)\right\}\right| 0\right\rangle=-\frac{\partial^{2} E_{v a c}(\theta)}{\partial \theta^{2}}=\frac{m_{q}}{N_{f}}\langle 0|\bar{\Psi} \Psi| 0\rangle+O\left(m_{q}^{2}\right) .
$$

Therefore, at small $\theta$ the vacuum energy is fixed $E(\theta)-E(0) \sim f_{\pi}^{2} m_{\pi}^{2} \theta^{2}$ and, thus the axion mass $m_{a}^{2}$ is also fixed: $V(a)-V(0) \sim f_{\pi}^{2} m_{\pi}^{2}\left(a / f_{a}\right)^{2}, m_{a}^{2} \sim f_{\pi}^{2} m_{\pi}^{2} / f_{a}^{2}$. As it should be, in the chiral limit the vacuum energy does not depend on $\theta$.

2. The vacuum energy $E_{v a c}(\theta)$ is a periodic function of $\theta$, i.e. $E_{v a c}(\theta \rightarrow 2 \pi+\theta)=E_{v a c}(\theta)$. This periodicity is a direct consequence of quantization of the topological charge in QCD:

$$
|\theta\rangle=\sum_{n} e^{i n \theta}|n\rangle, \quad|\theta+2 \pi\rangle \equiv|\theta\rangle
$$

where $|n\rangle$ is the winding state, $n$ is an integer. We note that the vacuum energy in gluodynamics (QCD without quarks) is also a $2 \pi$ periodic function.

3. In gluodynamics with the large number of colors $N_{c}$ the physics should depend on $\theta$ through the combination $\theta / N_{c}$ in order for the $\mathrm{U}(1)$ problem to be solved [11].

4. The anomalous WI's require [12] that Goldstone bosons which are described by the unitary matrix $U_{i j}$ should appear (apart from the mass term) only in the following combination with $\theta$ :

$$
\theta-i \operatorname{Tr} \log U
$$

This is the only allowed combination for arbitrary $N_{c}$ and $N_{f}$. This fact is a consequence of the transformation properties of the Goldstone fields $U \rightarrow \exp i \alpha U$ and the $\theta$ parameter $\theta \rightarrow \theta+N_{f} \alpha$ under the $U(1)$ chiral rotations. 


\section{Di Vecchia-Veneziano-Witten (VVW) Solution}

Based on the results outlined in the previous section, and also on the large $N_{c}$ arguments, Di Vecchia, Veneziano and Witten [12] suggested the following effective potential to analyze the $\theta$ dependence of $E_{v a c}(\theta)$ :

$$
W_{V V W}(\theta, U)=-\frac{\left\langle\nu^{2}\right\rangle_{Y M}}{2}(\theta-i \log \operatorname{Det} U)^{2}-\frac{1}{2} \operatorname{Tr}\left(M U+M^{+} U^{+}\right)+\ldots
$$

In this formula $M=\operatorname{diag}\left(m_{i}\left|\left\langle\bar{\Psi}^{i} \Psi^{i}\right\rangle\right|\right)$ and the coefficient in front of the combination $(\theta-i \log \operatorname{Det} U)^{2}$ is the topological susceptibility in pure YM theory rather than in real QCD: $\left\langle\nu^{2}\right\rangle_{Y M}=-\frac{\partial^{2} E_{Y M}}{\partial \theta^{2}}<0$.

The minimization of the potential $W_{V V W}(\theta, U)$ with respect to the $U$ fields gives the $\theta$ dependence of the vacuum energy. The obtained result has the required $2 \pi$ periodicity and actually is a more complicated function than the simple ansatz $\cos (\theta) \sim \cos \left(a / f_{a}\right)$ exploited in most calculations with the axions.

In spite of the great simplicity and attractiveness of the VVW solution, this scenario can not be quite complete. The argument is that pure YM theory, not only QCD, should also obey the $2 \pi$ periodicity law in $\theta$. For YM theory in the large $N_{c}$ limit the potential (田) would imply that the $\theta$ dependence in gluodynamics is given by $W_{Y M}(\theta)-W_{Y M}(\theta=$ $0)=-\left\langle\nu^{2}\right\rangle_{Y M} \theta^{2} / 2$, which can not be correct for an arbitrary $\theta$. The first guess would be that our failure to reproduce the $2 \pi$ periodicity is related to shortcomings of the $1 / N_{c}$ expansion: the next terms, suppressed by factor $1 / N_{c}$, presumably should recover the periodicity. However, this simple guess is not working. Indeed, any function like

$W_{Y M}(\theta)=-\frac{N_{c}^{2}\left\langle\nu^{2}\right\rangle_{Y M}}{2} \cos \left(\theta / N_{c}\right)$ which depends, as it should, on the combination $\theta / N_{c}$, would have period $2 \pi N_{c}$ rather than the required $2 \pi$. In addition, the approach of Ref.[12] deals from the very beginning with the light chiral degrees of freedom and explicitly incorporates the $U_{A}(1)$ anomaly without restriction of the topological charge to integer values.

Therefore, something is missing... To understand exactly what is missing, we should have a better understanding of gluodynamics and its periodic properties. Only after that we can come back to QCD. As the first step in this direction we would like to learn some lessons about the $\theta$ dependence which supersymmetric (SUSY) models offer to us.

\section{Lessons from SUSY theories}

In this section we would like to overview some SUSY models with an emphasize on the properties of vacuum states and their $\theta$ dependence. As we already learned in the case of QCD, this is the most relevant information for the axion physics. Instrumental for this analysis is the 'old-fashioned' effective Lagrangian approach, in which the effective Lagrangian is defined as the Legendre transform of the generating functional for connected Green functions. Only the potential part of this Lagrangian can be fixed in this way as it corresponds to zero momentum $n$-point correlation functions. The kinetic part is not fixed in this framework. Thus, such an effective Lagrangian is useless for calculating the $S$-matrix, but is perfectly suitable for addressing the vacuum properties of the theory. 
Fortunately, this is exactly the information we need: the $\theta$ dependence of the vacuum energy is the problem amenable to a study within this framework.

To be more specific, let us consider the effective Lagrangian for supersymmetric QCD (SQCD). The potential part of the Lagrangian, which is fixed by the anomalous Ward identities, is given by [13]:

$$
W_{S Q C D}=-\left.\frac{1}{3} S \ln \left(\frac{S^{N_{c}-N_{f}} \operatorname{Det} U}{\Lambda^{N_{c}} e^{-i \theta}}\right)\right|_{F}-\left.\operatorname{Tr}(m U)\right|_{F},
$$

where $S$ is the gauge chiral superfield, $\Lambda$ is the fundamental scale parameter of the theory, and $U_{j}^{i}=Q^{i} \tilde{Q}_{j}, i, j=1, . . N_{f}$ is the matter superfield with the mass matrix $m$. This Lagrangian should describe the vacuum structure of the theory. As was found in [14, there are $N_{c}$ different vacua in SQCD, which are labeled by the $\theta$ angle and the discrete parameter $k=0,1, \ldots N_{c}-1$ such that the gluino condensate depends on these parameters as $\langle\lambda \lambda\rangle \sim \exp \left(\frac{i \theta+2 \pi k}{N_{c}}\right)$. Therefore, when $\theta$ varies continuously from 0 to $2 \pi, N_{c}$ distinct and disconnected Bloch type vacua undergo a cyclic permutation: the first state becomes the second one, and so on. All physical quantities are periodic in $\theta$ with periodicity $2 \pi$, as these vacua can be just relabeled by the substitution $k \rightarrow k-1$ after the shift $\theta \rightarrow \theta+2 \pi$, keeping the physics intact [14].

Therefore, in SUSY theories we do have the property described in Sect.2: the $\theta$ dependence comes through the combination $\theta / N_{c}$, but the physics is $2 \pi$ periodic due to the existence of the additional states. In SUSY models they are degenerate, in nonsupersymmetric theories we expect that they have different energies.

Although the potential (5) has some appealing features, it can not be complete as was recently argued by Kovner and Shifman [8]. Indeed, the scalar potential (5) is not a single-valued function of the field. If one starts at some $S=S_{0}$ and travels continuously to the different (but physically equivalent) $S_{0}^{\prime}=e^{2 i \pi} S_{0}$, the value of the potential at $S_{0}^{\prime}$ will be different from that at $S_{0}$. Secondly, the discrete symmetry inherent to the original theory is not reflected in Eq.(5). Both these shortcomings were successfully cured in 8 by the prescription of summation over all branches of the multivalued potential (5). In the simplest case of supersymmetric gluodynamics $\left(N_{f}=0\right.$ in Eq.(5)), this prescription leads to the following definition of the effective potential $W_{K S}$ :

$$
\exp \left(-W_{K S}\right)=\sum_{n} \exp \left[\frac{1}{3} S \ln \left(\frac{S^{N_{c}}}{\Lambda^{N_{c}}}\right)+\text { h.c. }+\frac{1}{3}(S-\bar{S})(\theta+2 \pi n)\right],
$$

where for simplicity we suppressed the integrals over the superspace coordinates. In terms of the original theory, such prescription means the summation over all topological classes

$$
Z \sim \sum_{n} \int D A \exp \left[-S_{0}+i \int d^{4} x(\theta+2 \pi n) \frac{1}{32 \pi^{2}} G \tilde{G}\right]
$$

which imposes quantization of the topological charge $\int d^{4} x\left(1 / 32 \pi^{2}\right) G \tilde{G}$ in integer units. Therefore, the summation over all topological classes does not change our theory, but simply introduces an overall (infinite) factor into $Z$, which is irrelevant anyhow. It is clear that all anomalous Ward identities, as well as the dynamical part of the effective lagrangian (the first term in Eq.(可)) are kept intact by this prescription. 
It is not the purpose of this paper to discuss the technical details of this new development in gauge theories. We refer the reader to the original papers [8, 7] for details. Here we would like to end up this section by emphasizing some lessons to be learned from SUSY theories, which are relevant to us and important for the axion physics:

1. $\theta \rightarrow \theta+2 \pi$ is the explicit symmetry of the theory because it can be compensated for by a shift in $n \rightarrow n+1$ such that the potential (6) is unchanged.

2. The vacuum states are classified by two parameters $\left|\theta, k=0, . . N_{c}-1\right\rangle$. All these states are physically equivalent, but they are different vacuum states labeled by the condensate $\langle\lambda \lambda\rangle \sim \exp \left(\frac{i \theta+2 \pi k}{N_{c}}\right)$. This additional discrete quantum number $k$ can not be found from the analysis of the symmetries of the original lagrangian because of its quantum origin related to the anomaly. It can be understood only from explicit dynamical calculations.

3. In SUSY models these extra states are degenerate in energy. However, in nonsupersymmetric case one could generally expect that states with different energies (i.e. metastable vacua) appear for some values of $\theta$, which decay by tunneling. For the axion physics, this implies that decays of such metastable states may be supplemented by emission of the axions.

4. In general, there are domain walls in the system connecting different vacua. For the axion physics it would imply the existence of different kind of domain walls along with the ones studied previously [15].

\section{$5 \quad$ Effective Lagrangian and $\theta$ dependence in QCD}

In this section we describe the effective potential in QCD [7] which allows one to analyze the $\theta$ dependence of the ground state. In this approach, the Goldstone fields are described by the unitary matrix $U_{i j}$ corresponding to the $\gamma_{5}$ phases of the chiral condensate: $\left\langle\bar{\Psi}_{L}^{i} \Psi_{R}^{j}\right\rangle=-\left|\left\langle\bar{\Psi}_{L} \Psi_{R}\right\rangle\right| U_{i j}$ with

$$
U=\exp \left[i \sqrt{2} \frac{\pi^{a} \lambda^{a}}{f_{\pi}}+i \frac{2}{\sqrt{N_{f}}} \frac{\eta^{\prime}}{f_{\eta^{\prime}}}\right], U U^{+}=1,
$$

where $\lambda^{a}$ are the Gell-Mann matrices of $S U\left(N_{f}\right), \pi^{a}$ is the pseudoscalar octet, and $f_{\pi}=$ $133 \mathrm{MeV}$. In terms of $U$ and the "glueball" fields $h, \bar{h}$, the QCD effective potential can be constructed analogously to the SUSY case, and has the explicit $2 \pi$ periodicity:

$$
e^{-i V W(h, U)}=\sum_{n=-\infty}^{+\infty} \sum_{k=0}^{q-1} \exp \left[-i V W_{d}(h, U)+i \pi V\left(k+\frac{q}{p} \frac{\theta+2 \pi n}{2 \pi}\right) \frac{h-\bar{h}}{2 i}\right] .
$$

Here the "dynamical" part $W_{d}(h, U)$ of the anomalous effective potential is

$$
W_{d}(h, U)=\frac{1}{4} \frac{q}{p} h \log \left[\left(\frac{h}{2 e E}\right)^{p / q} \operatorname{Det} U\right]-\frac{1}{2} \operatorname{Tr} M U+h . c .
$$

where $V=\int d^{4} x$ is the 4 -volume. All dimensional parameters of this potential are expressed in terms of the QCD vacuum condensates, and are well known numerically: $M=\operatorname{diag}\left(m_{i}\left|\left\langle\bar{\Psi}^{i} \Psi^{i}\right\rangle\right|\right)$; the constant $E$ is related to the QCD gluon condensate $E=$ 
$\left\langle b \alpha_{s} /(32 \pi) G^{2}\right\rangle$. It is interesting to note that the whole structure of Eq.(9) is rather similar to that of the SUSY effective potential (6). Namely, it contains both the "dynamical" $W_{d}$ and "topological" parts (the first and the second terms in the exponent, respectively). The "dynamical" part of the effective potential (9) is similar to $W_{S Q C D}$ (5) while the "topological" part is akin to the improvement [8] of this effective potential.

The only unknown parameters in this construction are the integers $p, q$, which play the same role as discrete integer numbers in SUSY theories, see Sect.4. These numbers are related to a discrete symmetry which is a remnant of the anomaly, and can be found only by explicit dynamical calculations. One can argue 16 that $q / p=8 / 3 b$ where $b=(11 / 3) N_{c}-(2 / 3) N_{f}$ is the first coefficient of the $\beta$-function. However, for more generality we prefer not to fix $q / p$ in what follows with the only constraint that at large $N_{c}, \quad q / p \sim 1 / N_{c}$ in order for the $U(1)$ problem to be solved.

The heavy "glueball" fields $h, \bar{h}$ can be integrated out in Eq.(9). The resulting effective chiral potential is periodic in $\theta$ and takes the form [7]

$$
\begin{aligned}
W_{Q C D}\left(\theta, U, U^{+}\right)=-\lim _{V \rightarrow \infty} \frac{1}{V} \log \left\{\sum _ { l } \operatorname { e x p } \left[V E \cos \left[-\frac{q}{p}(\theta-i \log \operatorname{Det} U)+\frac{2 \pi}{p} l\right]\right.\right. \\
\left.\left.+\frac{1}{2} V \operatorname{Tr}\left(M U+M^{+} U^{+}\right)\right]\right\}, l=0,1, \ldots, p-1
\end{aligned}
$$

It was argued in [7] that Eq.(11) represents the anomalous effective Lagrangian realizing broken conformal and chiral symmetries of QCD. The arguments are the following: (a) Eq.( 11) correctly reproduces the VVW effective chiral lagrangian 12 in the large $N_{c}$ limit; (b) Eq.( 11) reproduces the anomalous conformal and chiral Ward identities of QCD.

(a) For small values of $(\theta-i \log \operatorname{Det} U)<\pi / q$, the term with $l=0$ dominates the infinite volume limit. Expanding the cosine (this corresponds to the expansion in $q / p \sim$ $1 / N_{c}$ ), we recover exactly the VVW effective potential (雨) at lowest order in $1 / N_{c}$, together with the constant term $-E=-\left\langle b \alpha_{s} /(32 \pi) G^{2}\right\rangle$ required by the conformal anomaly:

$$
W_{V V W}\left(\theta, U, U^{+}\right)=-E-\frac{\left\langle\nu^{2}\right\rangle_{Y M}}{2}(\theta-i \log \operatorname{Det} U)^{2}-\frac{1}{2} \operatorname{Tr}\left(M U+M^{+} U^{+}\right)+\ldots
$$

where we used the fact that at large $N_{c} E(q / p)^{2}=-\left\langle\nu^{2}\right\rangle_{Y M}$ is the topological susceptibility in pure YM theory. Corrections in $1 / N_{c}$ stemming from Eq.(11) constitute a new result.

(b) It is easy to check that the anomalous chiral and conformal WI's are reproduced by Eq.(11). As an important example, let us calculate the topological susceptibility in QCD near the chiral limit. For simplicity, we consider the limit of $S U\left(N_{f}\right)$ isospin symmetry with $N_{f}$ light quarks, $m_{i} \ll \Lambda_{Q C D}$. For the vacuum energy for $\theta<\pi / q$ we obtain

$$
E_{v a c}(\theta)=-E+m_{q} N_{f}\langle 0|\bar{\Psi} \Psi| 0\rangle \cos \left(\frac{\theta}{N_{f}}\right)
$$

Differentiating this expression twice in $\theta$, we reproduce the famous WI (11).

We note that in general (Eq.(13) is the particular example) the $\theta$ dependence comes in a combination $\theta / N$ which naively does not provide the desired $2 \pi$ periodicity for the 
physical observables. At the same time, this formula was derived from Eq.(11) which is perfectly $2 \pi$ periodic. How could it be? The answer is: the thermodynamic limit $V \rightarrow \infty$ is performed for a certain value of $\theta$, such that only a term of lowest energy survives in (11), while all other states have higher energies and therefore drop out. At the same time, the values $\theta$ and $\theta+2 \pi$ are physically equivalent for the whole set of states and not for a selected individual vacuum state. Thus, the fact that the $\theta$ dependence in usual $V=\infty$ formulas comes in the combination $\theta / N$ has nothing to do with the problem of periodicity in $\theta$, as those formulas refer to one particular state out of this set [7].

\section{Applications to the axion physics}

\subsection{Local properties of the axion potential}

Now we are ready to apply our results to the axion physics. The axion potential, by definition, is obtained from Eq.(11) by the replacement $\theta \rightarrow a / f_{a}$ :

$$
W\left(a, U, U^{+}\right) \equiv W_{Q C D}\left(\theta=a / f_{a}, U, U^{+}\right) .
$$

The next step is to integrate out the Goldstone fields $U$ exactly in the same way as was done in obtaining Eq.(13) for small values of $\theta$. Technically, this problem amounts to the minimization of the potential $W\left(a, U, U^{+}\right)$with respect to $U$ for a fixed value of $a$.

Before discussing the general global properties of this potential, we would like to emphasize that all parameters of the potential (except for the integers $p, q$ ) are fixed at zero temperature: $\langle\bar{\Psi} \Psi\rangle_{T=0} \simeq-(250 \mathrm{MeV})^{3} ; \quad\left\langle\alpha_{s} / \pi G^{2}\right\rangle_{T=0} \simeq 1.2 \cdot 10^{-2} \mathrm{GeV}^{4} ; \quad f_{\pi}(T=$ $0)=133 \mathrm{MeV}$. The dependence of these parameters on temperature is also (at least, qualitatively) known. In particular, the axion mass, which is defined as the quadratic coefficient in the expansion of the function $E_{v a c}(\theta)$ at small $\theta$, is proportional to the chiral condensate: $m_{a}^{2}(T) \sim m_{q}\langle 0|\bar{\Psi} \Psi| 0\rangle_{T} / f_{a}^{2}$. Therefore, $m_{a}^{2}(T)$ is known as long as $\langle 0|\bar{\Psi} \Psi| 0\rangle_{T}$ is known. This statement is exact up to the higher order corrections in $m_{q}$. We neglect these higher order corrections everywhere for $T \leq T_{c}\left(T_{c} \simeq 200 \mathrm{MeV}\right.$ is the critical temperature), where the chiral condensate is nonzero and gives the most important contribution to $m_{a}$. For the particular case $N_{f}=2$ one expects the second order phase transition and, therefore, $m_{a}^{2} \sim \frac{m_{q}}{f_{a}^{2}}\langle 0|\bar{\Psi} \Psi| 0\rangle \sim\left|T_{c}-T\right|^{\beta}$ for $T$ near $T_{c} \simeq 200 \mathrm{MeV}$. This is exactly where the axion mass does "turn on". The critical exponent in this case $\beta \simeq 0.38$, see e.g. recent reviews [17] for a general discussions of the QCD phase transitions.

In what follows we make the very plausible assumption that the temperature dependence for all observables for $T \leq T_{c}$ comes entirely through the parameters involved in the effective potential (the quark and gluon condensates and $f_{\pi}$ ). This assumption is based on the fact that the only relevant degrees of freedom at $T \leq T_{c}$ are the Goldstone bosons. With this assumption, the entire potential $W\left(a, U, U^{+}\right)_{T}$ for $T \leq T_{c}$ (not only the first term of its expansion $\sim m_{a}^{2}$ ) is also known. Therefore, the procedure described above allows us to construct the axion potential everywhere. In particular, for small fields $a$ in the limit of $S U\left(N_{f}\right)$ isospin symmetry with $N_{f}$ light quarks, the potential is:

$$
V(a)=-E+m_{q} N_{f}\langle 0|\bar{\Psi} \Psi| 0\rangle_{T} \cos \left(\frac{a}{f_{a} N_{f}}\right)+0\left(m_{q}^{2}\right), \quad \frac{a}{f_{a}} \ll \pi, \quad T \leq T_{c} .
$$


Up to now we discussed only the local properties of the axion potential. Its global (or topological) properties may be even more important for the axion physics.

\subsection{Global properties of the axion potential}

The topological properties of the potential crucially depend on the integer numbers $q, p$ we introduced earlier. Therefore, we consider the cases $q=1$ and $q \neq 1$ separately. We start from the simplest $q=1$ case. In this case the global properties of the potential qualitatively are similar to those of the VVW potential (4). The only difference from the latter is the presence of cusp singularities at certain values of the fields, which are a remnant of the topological charge quantization in the effective Lagrangian approach [7].

An interesting qualitative phenomenon which follows from the analysis of the potential (田) or (11) is a possible appearance of additional local minima at $\theta \sim \pi$, depending on temperature and quark masses. Therefore, the axion potential in this region may become a multi-valued function, i.e. there would be two different values of the axion potential $V_{1,2}\left(\theta=a / f_{a}\right)$ for a fixed $\theta$, which differ by the phase of the chiral field.

In particular, for the VVW potential (田) at $T=0$ for three flavors with equal masses and $\theta=\pi$, there are two degenerate states [12] separated by the domain wall. The wall surface tension in this case was recently calculated by Smilga [18]:

$$
\sigma=3 \sqrt{2}\left(1-\frac{\pi}{3 \sqrt{3}}\right) m_{\pi} f_{\pi}^{2}
$$

For $\theta \neq \pi$ the energies of the vacua are not degenerate anymore but are splitted apart by the amount $\Delta E \sim m_{q}(\theta-\pi)$. For the case of equal masses, a metastable state in the VVW scenario exists in the region $\pi / 2 \leq \theta \leq 3 \pi / 2$ [18]. (As we will discuss shortly, this phenomenon becomes far more general for the potential (11) if $q \neq 1$.)

A metastable vacuum decays to the ground state with the formation of bubbles of the stable phase. The quasiclassical formula for the decay rate per unit time per unit volume was derived many years ago [19:

$$
\Gamma \propto \exp \left(-\frac{27 \pi^{2} \sigma^{4}}{2(\Delta E)^{3}}\right) \equiv \exp \left(-S_{4}\right)
$$

Using (16), one finds that in the VVW scenario the lifetime of the metastable state at zero temperature is much larger than the age of the Universe [18]. Such phenomenon might play an important role in the development of the early Universe during the QCD epoch. However, to make the corresponding estimates one can not literally use Eq.(17) because the parameters which enter this formula depend on temperature and may drastically change the result near the phase transition point. It is quite possible that during the phase transition the relevant factor which enters (17) vanishes: $\sigma^{4} /(\Delta E)^{3} \sim\left|T_{c}-T\right|^{\alpha} \rightarrow 0, \alpha>$ 0 at $\left|T_{c}-T\right| \rightarrow 0$ and, therefore, $\Gamma \sim 1$. Indeed, in the mean field approximation where $\langle 0|\bar{\Psi} \Psi| 0\rangle_{T} \sim\left(T_{c}-T\right)^{1 / 2}, \quad f_{\pi}^{2} \sim\left(T_{c}-T\right)$ we have $\sigma \sim\left(T_{c}-T\right)^{3 / 4}$ and $\Delta E \sim\left(T_{c}-T\right)^{1 / 2}$. Therefore $\sigma^{4} /(\Delta E)^{3} \sim\left|T_{c}-T\right|^{3 / 2} \rightarrow 0$.

\footnotetext{
${ }^{1}$ This formula is derived for zero temperature, for a very high temperature [20] the correct expression is $\Gamma \propto \exp \left\{-16 \pi \sigma^{3} /\left(3 T(\Delta E)^{2}\right)\right\}$.
} 
Now we assume that the axion exists. In this case, if the axion field at temperature $T \simeq T_{c}$ is trapped in a metastable minimum at $\theta \sim \pi$, there are two different options for the decay of this false vacuum. If $\Gamma \sim 1$ at $T \simeq T_{c}$, tunneling is not suppressed, and the false vacuum tends to lower its energy first by bubble nucleation without changing $\theta$, and then by relaxing to $\theta=0$ with production of axions. This is advantageous since the axion potential is nearly flat. However, if $\Gamma$ is still very small at $T \simeq T_{c}$, the main mechanism for the decay could be related to the direct axion production.

For the case $q \neq 1$ (which we prefer, see [16]), the physics is even more interesting. Additional metastable vacua appear for arbitrary quark masses and for an arbitrary $\theta$, not necessarily in the region $\theta \sim \pi$. Therefore, decays of these false vacuum states will be a general phenomenon. In this case the wall surface tension can be easily calculated from (11) and for $\theta=0$ is given by (see [21] for details)

$$
\sigma=\frac{4 p}{q \sqrt{N_{f}}} f_{\pi} \sqrt{\left\langle\frac{b \alpha_{s}}{32 \pi} G^{2}\right\rangle}\left(1-\cos \frac{\pi}{2 p}\right)+0\left(m_{q} f_{\pi}^{2}\right) .
$$

This formula substitutes Eq.(16) describing the wall surface tension at $\theta \simeq \pi$ for the VVW potential (团). A distinct difference between these two cases is the absence of the chiral suppression $\sim m_{q}$ in Eq.(18), which apparently would make penetration through the barrier even more difficult in comparison to the VVW potential. The energy splitting between the ground state and metastable state at $\theta=0$ is

$$
\Delta E=m_{q} N_{f}|\langle\bar{\Psi} \Psi\rangle|\left(1-\cos \frac{2 \pi}{q N_{f}}\right)+0\left(m_{q}^{2}\right),
$$

and we obtain

$$
S_{4}=\frac{3^{3} \cdot 2^{7} \cdot \pi^{2} p^{4}}{q^{4} N_{f}^{5}} \frac{f_{\pi}^{4} E^{2}}{M^{3}} \frac{\left(1-\cos \frac{\pi}{2 p}\right)^{4}}{\left(1-\cos \frac{2 \pi}{q N_{f}}\right)^{3}} \simeq \frac{27}{256} \frac{\pi^{4} q^{2} N_{f}}{p^{4}} \frac{f_{\pi}^{4}\left\langle\frac{b \alpha_{s}}{32 \pi} G^{2}\right\rangle^{2}}{m_{q}^{3}|\langle\bar{\Psi} \Psi\rangle|^{3}} .
$$

Eq.(20) shows that the parametric suppression of the decay is largely overcome due to a numerical enhancement. The latter depends crucially on the particular values of the integers $p, q$. In particular, for our favorite choice $p=11 N_{c}-2 N_{f}, q=8$ [16], Eq.(20) yields a factor $\simeq 10$, while e.g. for $p=N_{c}, q=1$ (as motivated by SUSY, see [21]) it is approximately two orders of magnitude larger, but still much smaller than the estimate of 18 for the VVW potential. Of course, all remarks made above concerning the temperature dependence of such effect apply for the estimate (20) as well. In particular, $\Gamma$ could be of order one during the QCD phase transition in development of the early Universe and, therefore, a metastable state could decay just before the Universe cools down to the temperature where Eq. (20) could be applied.

Another related question is the dynamics of the domain walls which separate two different vacua with the small splitting $\Delta E$ (19). These domain walls are absolutely harmless for development of our universe because they decay in proper time scale. Indeed, the required value for the pressure (which is equal to $\Delta E$ in our notations) for the safe decay of the wall is given by 22]

$$
\Delta E=m_{q} N_{f}|\langle\bar{\Psi} \Psi\rangle|\left(1-\cos \frac{2 \pi}{q N_{f}}\right) \geq \frac{\sigma^{2}}{M_{P}^{2}},
$$


where $M_{P}$ is the Plank scale. Inequality (21) is perfectly satisfied with our parameters $\Delta E$ and $\sigma$ (18). Therefore, such domain walls do not lead to a cosmological disaster, but rather may have very interesting cosmological consequences which still have to be explored. Similar domain walls which separate vacua with different phases of the gluino condensate have been recently discussed by Shifman in SUSY models [23]. It is quite remarkable that this phenomenon may exist not only in SUSY models but also in the physically relevant case of QCD.

We conclude this section by emphasizing that the phenomena just described may lead to the necessity to reconsider the constraints on a dark matter axion because the dynamics of the axion field could be more complicated than it was originally thought. We stress that the decay of the metastable state described above proceeds (with or without axions) through the expansion of bubbles with $100 \%$ violation of CP invariance. This is because the phase of the chiral condensate in the metastable vacuum is nonzero and of order 1 . This leads to violation of $\mathrm{CP}$ even if $\theta=0$. (This is not at variance with the VafaWitten theorem [24] which refers to the lowest energy state only.) It may have profound consequences for the development of the early Universe at the QCD scale because such effects could lead to a new mechanism for baryogenesis! Indeed, the famous Sakharov criteria [9] could be satisfied in this scenario, see Sect. 8 for a more detailed discussion.

\section{$7 \quad$ New axion search experiment at RHIC?}

The development of the early Universe is a remarkable laboratory for the study of most nontrivial properties of the particle physics. What is more amazing is the fact that these phenomena at the QCD scale can be, in principle, experimentally tested at RHIC, Brookhaven.

We expect that, in general, an arbitrary $|\theta\rangle$-state would be created in the heavy ion collisions, similarly to the creation of the disoriented chiral condensate (DCC) with an arbitrary isospin direction. It should be a large domain with a wrong $\theta \neq 0$ orientation. As we shall see in a moment, for both cases (DCC and $|\theta\rangle$-state) the difference in energy between a created state and the lowest energy state is proportional to $m_{q}$ and negligible at high temperature. Therefore, energetically an arbitrary $|\theta\rangle$ can be formed. The way of how the created $|\theta\rangle$-state will relax to the ground state of lowest energy $|\theta=0\rangle$ is a separate issue. If, somehow, an equilibrium state with a large correlation length in the large volume $V$ is formed, the only possible way to relax $\theta$ to zero would be the axion mechanism (if they exist). However, due to the fact that we do not expect to create an equilibrium state with an infinite correlation length in the heavy ion collisions, the decay of a $|\theta\rangle$-state will also occur due to the Goldstone $U$ fields with specific $C P$-odd correlations. Therefore, two mechanisms of the relaxation of a $|\theta\rangle$-state to the vacuum would compete: the axion one and the standard decay to the Goldstone bosons. In the large volume limit if a reasonably good equilibrium state with a large correlation length is created, the axion mechanism would win; otherwise, the Goldstone mechanism would win. In any case, the result of the decay of a $|\theta\rangle$-state would be very different depending on the presence or absence of the axion field in Nature.

\footnotetext{
${ }^{2}$ A similar phenomenon has been recently discussed in Ref. 25.
} 
Before going into details, we would like to recall some general properties of the DCC which (hopefully) can be produced at RHIC (see e.g. [26] for a review), with an emphasize on the analogy between the DCC and a misaligned $|\theta\rangle$-state. If the cooling process is very rapid and, therefore, the system is out of equilibrium, there will be a large size of the correlated region in which the vacuum condensate orientation mismatches its zero temperature value. The absolute value of the chiral condensate right after the phase transition is expected to be close to its final (zero temperature) magnitude. However the vacuum direction of the formed condensate is still misaligned since it takes a longer time for the vacuum orientation to relax due to the small free energy difference $\sim m_{q}$ between the formed and true vacuum states.

To be more specific, let us consider the case $N_{f}=2$. The matrix $U$ is parametrized by the misalignment angle $\phi$ and the unit vector $\vec{n}$ in the isospin space:

$$
U=e^{i \phi(\vec{n} \vec{\tau})}, \operatorname{Tr}\left(\tau^{a} \tau^{b}\right)=2 \delta^{a b}, U U^{+}=1,\left\langle\bar{\Psi}_{L}^{i} \Psi_{R}^{j}\right\rangle=-\left|\left\langle\bar{\Psi}_{L} \Psi_{R}\right\rangle\right| U_{i j}
$$

The energy density of the DCC is determined by the mass term:

$$
E_{\phi}=-\frac{1}{2} \operatorname{Tr}\left(M U+M^{+} U^{+}\right)=-2 m|\langle\bar{\Psi} \Psi\rangle| \cos (\phi)
$$

where we put $m_{u}=m_{d}=m$ for simplicity. Eq.(23) implies that any $\phi \neq 0(\bmod 2 \pi)$ is not a stable vacuum state because $\left.\frac{\partial E_{\phi}}{\partial \phi}\right|_{\phi \neq 0} \neq 0$, i.e. the vacuum is misaligned. On the other hand, the energy difference between the misaligned state and true vacuum with $\phi=0$ is small and proportional to $m_{q}$. Therefore, the probability to create a state with an arbitrary $\phi$ at high temperature $T \sim T_{c}$ is proportional to $\exp \left[-V\left(E_{\phi}-E_{0}\right) / T\right]$ and depends on $\phi$ only very weakly, i.e. $\phi$ is a quasi-flat direction. Right after the phase transition when $\langle\bar{\Psi} \Psi\rangle$ becomes nonzero, the pion field begins to roll toward $\phi=0$, and of course overshoots $\phi=0$. Thereafter, $\phi$ oscillates. One should expect the coherent oscillations of the $\pi$ meson field which would correspond to a zero-momentum condensate of pions. Eventually these classical oscillations produce real $\pi$ mesons which hopefully can be observed. In a sense this picture is very similar to the standard "misalignment" mechanism [5, 3] for production of the cosmic axions during the QCD epoch.

Now we turn to our main point when the $U(1)_{A}$ phase of the disoriented chiral condensate is also nonzero and, therefore, the $|\theta\rangle$-vacuum state could be formed. To take into account this $U(1)_{A}$ phase we choose the matrix $U_{i j}$ in the form $U=\operatorname{diag}\left(e^{i \phi_{i}}\right)$. The energy density of the misaligned vacuum is determined in this case by Eq.(11). The most important difference between Eqs. (23) and (11) is the presence of the parametrically large term $\sim E \gg m_{q}|\langle\bar{\Psi} \Psi\rangle|$ in the expression for energy (11), describing the $U(1)_{A}$ phase of the disoriented chiral condensate. This term, as was explained in Sect.5, is related to the anomalous WI's, and does not go away in the chiral limit.

The key point is the following. For arbitrary phases $\phi_{i}$ the energy of a misaligned state differs by a huge amount $\sim E$ from the vacuum energy. Therefore, apparently there are no quasi-flat directions along $\phi_{i}$ coordinates, which would lead to the long wavelength oscillations with production of a large size domain. However, when the relevant combination $\left(\sum_{i} \phi_{i}-\theta\right)$ from Eq.(11) is close by an amount $\sim O\left(m_{q}\right)$ to its vacuum value, a Boltzmann suppression due to the term $\sim E$ is absent, and an arbitrary misaligned $|\theta\rangle$ state can be formed. In this case for any $\theta$ the difference in energy between the true $|\theta\rangle$ 
vacuum and a misaligned $|\theta\rangle$ - state (when the $\phi_{i}$ fields are not yet in their final positions $\left.\overline{\phi_{i}(\theta)}\right)$ is proportional to $m_{q}$ and very small in close analogy to the DCC case.

After this point we can apply the same philosophy as for DCC. The chiral fields $\phi_{i}$ begin to roll toward the true solution $\overline{\phi_{i}(\theta)}$ and of course overshoots it. The situation is very similar to what was described for the DCC with the only difference that in general we expect an arbitrary $|\theta\rangle$-disoriented state to be created in heavy ion collisions, not necessarily the $|\theta=0\rangle$ state. The difference in energy between these states is proportional to $m_{q}$, as follows from the fact that the $\theta$ dependence of any physical observable is proportional to $m_{q}$.

If a reasonably stable $|\theta \neq 0\rangle$ state is created, it could decay into the axions. To estimate the effect, we consider an ideal case when all energy stored in the $|\theta \neq 0\rangle$ state will be released through production of the axions. In this case from Eq.(13) we expect the following axion density $n_{a}$ for a random parameter $\theta \sim 1$ :

$$
n_{a}=\rho \frac{m_{q} N_{f}}{m_{a}}|\langle\bar{\Psi} \Psi\rangle|\left(1-\cos \frac{\theta}{N_{f}}\right) \sim \rho \cdot 10^{19} M_{e V} V^{3}\left(\frac{10^{-5} e V}{m_{a}}\right),
$$

where parameter $\rho$ is a suppression factor, see footnote (5). The standard way to detect the produced axions is to use their property of conversion to photons in an external magnetic field [28]. The relevant axion-photon coupling constant is defined in the following way:

$$
L_{a \gamma \gamma}=\frac{g_{a \gamma \gamma}}{4} a \tilde{F_{\mu \nu}} F_{\mu \nu}, \quad g_{a \gamma \gamma}(D F S Z)=\frac{\alpha}{2 \pi f_{a}} \quad g_{a \gamma \gamma}(K S V Z)=-\frac{\alpha}{2 \pi f_{a}} \cdot \frac{5}{3}
$$

where we specified the coupling constants in the limit $m_{u}=m_{d}$ for two popular models [2]. The conversion probability is given by [28]:

$$
P_{a \rightarrow \gamma}=\frac{1}{4} g_{a \gamma \gamma}^{2}\left(B^{2} l^{2}\right)
$$

where $B$ is the external magnetic field and $l$ its length. With our estimate (24) for the axion density, one can get the following formula for the probability of detection of a photon from the axion conversion [27]:

$$
P_{\gamma} \sim P_{a \rightarrow \gamma} n_{a} V \sim \rho m_{a}\left(\frac{\alpha}{2 \pi}\right)^{2}\left(B^{2} l^{2}\right) V
$$

where we took into account the relation $f_{a}^{2} m_{a}^{2} \sim m_{q}\langle 0|\bar{\Psi} \Psi| 0\rangle$, see Sect.6.1. In this formula $V \sim \lambda^{3}$ where $\lambda$ is the correlation length for the misaligned $|\theta\rangle$-state. As was discussed

\footnotetext{
${ }^{3}$ If $\theta \neq 0$, the Goldstone fields are not exactly the pseudoscalar fields, but rather are mixed with the scalars; the mixing angle between the singlet and octet combinations also depends on $\theta$, see [21] for detail.

${ }^{4}$ The possibility of a production of the axions in the heavy ion collisions was independently discussed by Melissinos [27]. AZ thanks Adrian Melissinos for a conversation on the subject.

${ }^{5}$ We are, of course, aware that this ideal case can not be realized in Nature. There should be a strong suppression factor $\rho$ in front of Eq.(24) due to the fact that there is no superselection rule in a finite volume nonequilibrium state. Therefore, the $\theta$ parameter can be relaxed to zero by others means, not necessarily related to the axion productions. The parameter $\rho$ should depend, first of all, on the correlation length $\lambda$ of the formed misaligned $|\theta\rangle$ state. We do not know at the moment how to estimate this parameter $\rho(\lambda)$. For the ideal case, $\rho=1$.
} 
above, the mechanism of production of axions by the coherent processes corresponds to a condensate of the "almost" zero-momentum $\left(\sim m_{q} / \lambda\right)$ axions. If the collision is asymmetric (as was suggested in [27]), the photon signal from the axion-photon conversion will be unambiguous because it will be the "almost" monochromatic wave with $\Delta \omega \sim$ $m_{q} / \lambda$ and $\omega$ to be determined by the asymmetry in energies of the beams.

Therefore, the heavy ion collisions give us a unique chance for a new axion search experiment. We would like to stop here with this optimistic note.

\section{Conclusion}

- Probably the most important outcome of our study is a better understanding of the global and local properties of the axion potential $V(a)$, which may result in the necessity to reconsider the constraints on a dark matter axion. The temperature dependence of the potential at $T \leq T_{c}$ is expressed in terms of the $T$-dependent vacuum condensates which have been extensively studied on the lattice and in the models [17] and are fairly known. Therefore, we know the temperature dependence of the potential as long as we know the temperature dependence of the chiral and gluon condensates.

- The axion potential is generically a multi-valued function. Such a property may lead to the phenomenon of the false vacuum decay through bubble nucleation, which may make the dynamics of the axion field far more complicated (and interesting) than was previously thought.

- Based on the analysis of the potential $V(a)$ expressed in terms of the condensates and the knowledge of their temperature dependence, we suggested a new idea for the axion search experiment at RHIC.

- As a byproduct of our analysis (which was mentioned at the end of Sect.6), we would like to speculate on the possibility of baryogenesis at the QCD scale (with or without axions). Indeed, it appears that all three famous Sakharov criteria [9] could be satisfied in the decay of a metastable state discussed above:

1. Such a metastable state is clearly out of equilibrium;

2. CP violation is unsuppressed and proportional to $m_{u} m_{d} m_{s} \bar{\theta}_{\text {eff }}, \bar{\theta}_{\text {eff }} \sim 1$. As is known, this is the most difficult part to satisfy in the scenario of baryogenesis at the electroweak scale within the standard model for $\mathrm{CP}$ violation;

3. The third Sakharov criterion is violation of the baryon $(B)$ number. Of course, the corresponding $\mathrm{U}(1)$ is an exact global symmetry of QCD. However, a "spontaneous" breaking of the baryon $\mathrm{U}(1)$ symmetry (as a result of interactions with the domain wall) is not forbidden, and would be sufficient. The latter may arise in a way similar to electroweak baryogenesis, see e.g. [29] for a review.

The viability and details of such a mechanism for baryogenesis are still to be explored [30, however, in general, one could expect a large asymmetry according to the unsuppressed CP violation $\sim m_{u} m_{d} m_{s} / \Lambda^{3}$ in bubble nucleation. What is amazing is the fact that each step in such a scenario for baryogenesis could be, in principle, experimentally tested at RHIC, Brookhaven.

- The remark that QCD scale could be an interesting place to look is also motivated by recent observations of the MACHO collaboration [31]. In particular, the domain walls 
with QCD scale which always separate the true and false vacuum states do not lead to a cosmological disaster, see (21). They rather may be very interesting objects for the problem of the structure formation at small scales $\sim M_{\odot}$.

\section{References}

[1] R. Peccei and H. Quinn, Phys. Rev. Lett. 38 (1977) 1440; S. Weinberg, Phys. Rev. Lett. 40 (1978) 223; F. Wilczek, Phys. Rev. Lett. 40 (1978) 279.

[2] J.E. Kim, Phys. Rev. Lett. 43 (1979) 103; M.A. Shifman, A.I. Vainshtein, and V.I. Zakharov, Nucl. Phys. B166 (1980) 493 (KSVZ).

M. Dine, W. Fischler, and M. Srednicki, Phys. Lett. B104 (1981) 199; A.R. Zhitnitsky, Yad.Fiz. 31 (1980) 497 (Sov. J. Nucl. Phys. 31 (1980) 260) (DFSZ).

[3] J.E. Kim, Phys. Rep. 150 (1987) 1; H.Y. Cheng, Phys. Rep. 158 (1988) 1; R.D. Peccei, in "CP violation", ed. C. Jarlskog, World Scientific, Singapore, 1989.

[4] R. Davis, Phys. Rev. D 32 (1985) 3172; R. Davis and E.P.S. Shellard, Nucl. Phys. B 324 (1989), 167; E.P.S. Shellard and R. A. Battye, astro-ph/9802216.

D.Harari and P. Sikivie, Phys. Lett. B195, (1987) 361; C.Hagmann and P. Sikivie, Nucl. Phys. B 363 (1991) 247.

[5] J. Preskil, M. Wise and F. Wilczek, Phys. Lett. B120 (1983) 127; L Abbott and P. Sikivie, Phys. Lett. B120 (1983) 133; M.Dine and W. Fischler, Phys. Lett. B120 (1983) 137.

[6] M.S. Turner, Phys. Rev. D 33 (1986) 889.

[7] I. Halperin and A. Zhitnitsky, hep-ph/9711398, to be published in Phys. Rev. D; hep-ph/9803301, submitted to Phys. Rev. Lett.

[8] A. Kovner and M. Shifman, Phys. Rev. D56 (1997) 2396 (hep-th/9702174).

[9] A.D. Sakharov, JETP Lett. 5 (1967) 24.

[10] R.J. Crewther, Phys. Lett. 70B (1977) 349.

[11] E. Witten, Nucl. Phys. B156 (1979) 269; G. Veneziano, Nucl. Phys. B159 (1979) 213.

[12] E. Witten, Ann. Phys. 128 (1980) 363; P. Di Vecchia and G. Veneziano, Nucl. Phys. B171 (1980) 253.

[13] G. Veneziano and S. Yankielowicz, Phys. Lett. B113 (1982) 231; T. Taylor, G. Veneziano and S. Yankielowicz, Nucl. Phys. B218 (1983) 439.

[14] M.A. Shifman and A.I. Vainshtein, Nucl. Phys. B296 (1988) 445. 
[15] A. Vilenkin and A. Everett, Phys. Rev. Lett. 48 (1982) 1867; P.Sikivie, Phys. Rev. Lett. 48 (1982) 1156; D. Lyth and E. Stewart, Phys. Rev. D 46 (1992) 532.

[16] I. Halperin and A. Zhitnitsky, hep-th/9802095; hep-ph/9707286.

[17] T. Schafer and E. V. Shuryak, Rev. Mod. Phys. 70 (1998) 323; A. Smilga, Phys. Rep. 291 (1997) 1.

[18] A. Smilga, hep-ph/9805214.

[19] M.B. Voloshin, I.Yu. Kobzarev and L.B. Okun, Sov. J. Nucl. Phys. 20 (1975) 644; S. Coleman, Phys. Rev. D15 (1977) 2929.

[20] A.D. Linde, Phys. Lett. 70B (1977) 306; Nucl. Phys. B216 (1983) 321.

[21] T. Fugleberg, I. Halperin and A. Zhitnitsky, to appear.

[22] A. Vilenkin, Phys. Rev. D23 (1981) 852.

[23] M. Shifman, hep-th/9704114.

[24] C. Vafa and E. Witten, Phys. Rev. Lett. 53 (1984) 535.

[25] D. Kharzeev, R. Pisarski, M. Tytgat, hep-ph/9804221.

[26] K. Rajagopal, hep-ph/9504310.

[27] A.C. Melissinos, hep-ph/9805507.

[28] P. Sikivie, Phys. Rev. Lett. 51 (1983) 1415, Phys. Rev. Lett. 52 (1984) 695; G. Rafelt and L. Stodolsky, Phys. Rev. D37 (1988) 1237.

[29] V.A. Rubakov and M.E. Shaposhnikov, Usp. Fiz. Nauk 166 (1996) 493 hepph/9603208); A.D. Dolgov, hep-ph/9707419; M. Trodden, hep-ph/9803479.

[30] R. Brandenberger, I. Halperin and A. Zhitnitsky, work in progress.

[31] K. Jedamzik, astro-ph/9805147. 